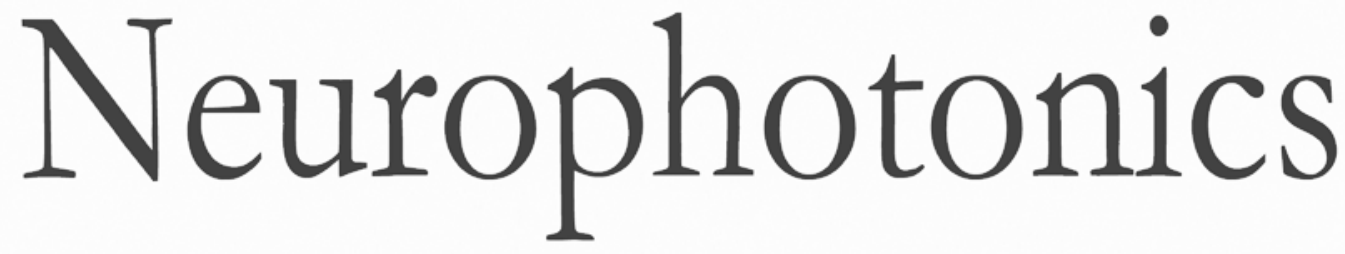

\title{
Selective photobiomodulation for emotion regulation: model-based dosimetry study
}

Paolo Cassano

Anh Phong Tran

Husam Katnani

Benjamin S. Bleier

Michael R. Hamblin

Yaoshen Yuan

Qianqian Fang 


\title{
Selective photobiomodulation for emotion regulation: model-based dosimetry study
}

\author{
Paolo Cassano, ${ }^{a, b, \dagger}$ Anh Phong Tran, ${ }^{c, \dagger}{ }^{\dagger}$ Husam Katnani, ${ }^{d}$ Benjamin S. Bleier, ${ }^{e}$ Michael R. Hamblin, ${ }^{f, g}$ \\ Yaoshen Yuan, ${ }^{h}$ and Qianqian Fang ${ }^{i, *}$ \\ ${ }^{a}$ Massachusetts General Hospital, Depression Clinical and Research Program, Center for Anxiety and Traumatic Stress Disorders, Boston, \\ Massachusetts, United States

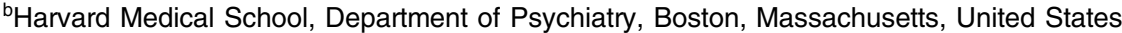 \\ 'Northeastern University, Department of Chemical Engineering, Boston, Massachusetts, United States \\ 'Massachusetts General Hospital, Harvard Medical School, Department of Neurosurgery, Boston, Massachusetts, United States \\ 'Massachusetts Eye and Ear Infirmary, Harvard Medical School, Department of Otolaryngology, Boston, Massachusetts, United States \\ ${ }^{f}$ Massachusetts General Hospital, Wellman Center for Photomedicine, Boston, Massachusetts, United States \\ gHarvard Medical School, Department of Dermatology, Boston, Massachusetts, United States \\ ${ }^{h}$ Northeastern University, Department of Electrical and Computer Engineering, Boston, Massachusetts, United States \\ 'Northeastern University, Department of Bioengineering, Boston, Massachusetts, United States
}

\begin{abstract}
The transcranial photobiomodulation (t-PBM) technique is a promising approach for the treatment of a wide range of neuropsychiatric disorders, including disorders characterized by poor regulation of emotion such as major depressive disorder (MDD). We examine various approaches to deliver red and near-infrared light to the dorsolateral prefrontal cortex (dIPFC) and ventromedial prefrontal cortex (vmPFC) in the human brain, both of which have shown strong relevance to the treatment of MDD. We apply our hardware-accelerated Monte Carlo simulations to systematically investigate the light penetration profiles using a standard adult brain atlas. To better deliver light to these regions-of-interest, we study, in particular, intranasal and transcranial illumination approaches. We find that transcranial illumination at the F3-F4 location (based on 10-20 system) provides excellent light delivery to the dIPFC, while a light source located in close proximity to the cribriform plate is well-suited for reaching the vmPFC, despite the fact that accessing the latter location may require a minimally invasive approach. Alternative noninvasive illumination strategies for reaching vmPFC are also studied and both transcranial illumination at the Fp1-FpZ-Fp2 location and intranasal illumination in the mid-nose region are shown to be valid. Different illumination wavelengths, ranging from 670 to $1064 \mathrm{~nm}$, are studied and the amounts of light energy deposited to a wide range of brain regions are quantitatively compared. We find that $810 \mathrm{~nm}$ provided the overall highest energy delivery to the targeted regions. Although our simulations carried out on locations and wavelengths are not designed to be exhaustive, the proposed illumination strategies inform the design of t-PBM systems likely to improve brain emotion regulation, both in clinical research and practice. ( ) The Authors. Published by SPIE under a Creative Commons Attribution 4.0 Unported License. Distribution or reproduction of this work in whole or in part requires full attribution of the original publication, including its DOI. [DOI: 10.1117/1.NPh.6.1.015004]
\end{abstract}

Keywords: photobiomodulation; near-infrared radiation; low-level light therapy; near-infrared laser transmission; transcranial light therapy; intranasal light therapy.

Paper 18056R received Sep. 26, 2018; accepted for publication Jan. 7, 2019; published online Feb. 7, 2019.

\section{Introduction}

Brain photobiomodulation (PBM) with near-infrared (NIR) and red light is a growing field of research. ${ }^{1,2}$ Over-the-counter lightemitting diodes (LEDs), as well as in-office laser devices, are already used off-label for transcranial photobiomodulation $(\mathrm{t}-\mathrm{PBM})$ in patients with neuropsychiatric disorders. ${ }^{3}$ Given the off-label use of t-PBM, only patients who did not respond to, or did not tolerate FDA-approved (or evidence-based) treatments are typically referred to t-PBM. The use of t-PBM devices is expected to increase as several studies are being conducted to test the efficacy and tolerability of t-PBM for neuropsychiatric disorders; specific FDA indications for the use of t-PBM might also arise. A wide range of on-going or concluded t-PBM clinical trials, including those targeting major depressive disorder (MDD) (NCT02898233, NCT02959307), generalized anxiety disorder (NCT03420456), dementia (NCT03160027), and

*Address all correspondence to Qianqian Fang, E-mail: q.fang@ neu.edu

†These authors contributed equally to this work. traumatic brain injury (TBI) with posttraumatic stress disorder (NCT02356861), have been reported in public databases. ${ }^{4}$

This relatively broad range of neuropsychiatric disorders, on which PBM is currently being tested, is justified by the equally broad proposed range of mechanisms of action of PBM. NIR and red light are absorbed by cytochrome $\mathrm{C}$ oxidase and stimulate the mitochondrial respiratory chain leading to increased adenosine triphosphate production. ${ }^{5-7}$ NIR can induce short bursts of reactive oxygen species leading to the activation of antioxidant mechanisms resulting in reduction of oxidative stress. Anti-inflammatory effects have also been demonstrated: NIR $(810 \mathrm{~nm})$ decreased production of TNF- $\alpha$, IL- $1 \beta$, and IL- $8 .{ }^{8}$ Light with the same wavelength $(810 \mathrm{~nm})$ decreased cellular infiltration in a rat model of spinal cord injury. ${ }^{9}$ Moreover, transcranial NIR $(800 \mathrm{~nm})$ reduced neuroinflammation in mice models of TBI. ${ }^{10} \mathrm{PBM}$ also stimulates neurogenesis and protects against cell death. Animal research has shown that NIR improves neurogenesis and synaptogenesis, via increase of brain derived neurotrophic factor. ${ }^{11,12}$ Other neurotrophic mechanisms have also been proposed for PBM such as the inhibition 
of glycogen synthase kinase 3 beta, otherwise known as GSK-3$\beta$, and of proapoptotic molecules. ${ }^{13}$

Preliminary clinical evidence on the efficacy of t-PBM for neuropsychiatric disorders is quite encouraging; however, as exemplified by the studies on t-PBM for affective disorders, there are methodological limitations preventing definite conclusions. In an open study, 10 patients with treatment resistant depression were treated with a single session of t-PBM $(810 \mathrm{~nm})$ at two sites on the forehead, using an LED instrument; the response rate of MDD at week 2 was $40 \% .{ }^{14}$ A second open study reported the results of the treatment with six sessions of t-PBM $(808 \mathrm{~nm})$ in four participants with moderate to severe MDD; two patients responded, respectively, at weeks 6 and 7. ${ }^{15}$ A randomized controlled trial (RCT) assessed the additive effect of t-PBM $(1064 \mathrm{~nm})$ onto attention bias modification (ABM) in individuals $(n=51)$ with elevated depressive symptoms. In those who responded to the ABM, the improvement was enhanced by the right t-PBM. ${ }^{16}$ An RCT designed to assess the efficacy of t-PBM $(823 \mathrm{~nm})$ as a primary treatment for MDD included 21 participants randomized to receive bilateral stimulation on the forehead (real or sham) twice-a-week for eight weeks. ${ }^{17}$ The decrease in depression severity was significantly greater in the PBM group compared to the sham. Response was observed in $50 \%$ of those who receive the active treatment and in $27 \%$ of those in the sham group. Noticeably, in several of the studies, the therapeutic benefits were not sustained when treatment was withdrawn. ${ }^{14,15}$

While there is growing evidence for the therapeutic value of t-PBM, many key questions related to the optimization of t-PBM devices and protocols remain unanswered, among which, a key question is: how to deliver most effectively NIR and red light to the brain? For instance, it is possible that some neuropsychiatric disorders may respond better to selective or preferential targeting of specific brain regions. ${ }^{16,18}$ While the literature in humans has addressed important questions concerning the penetration of t-PBM, such as the effects of the light source (LED versus laser), ${ }^{19}$ wavelength (NIR and red light) ${ }^{20,21}$ pulsing, ${ }^{22}$ skull thickness, and absorption of the light by blood, ${ }^{20}$ it remains to be determined what is the best placement(s) of the light source-both on the scalp and within cranial cavities- to selectively or preferentially target light to specific regions of the brain.

This paper does not directly address the question of the most therapeutic modality of delivery, but rather it answers the question of how to selectively target clinically relevant brain regions. Multiple, specific positions of the light source were studied, for both transcranial and intranasal (t-PBM and i-PBM) light delivery. Selective targeting of specific brain regions requires knowing the amount of light deposited on the targeted brain region, on the brain between that and the light source, and to other adjacent regions. A ratio of one or more orders of magnitude in energy deposition between the index cortex (10-fold higher) and other brain regions is considered sufficient for selective targeting, as a threshold amount of therapeutic energy could be exclusively deposited at the target cortex, by appropriately calibrating power and exposure time of the light source. We narrowed down the focus of this paper to brain regions that are both easily accessible and known to contribute to emotion regulation: specifically, the dorsolateral prefrontal cortex (dlPFC) and ventromedial prefrontal cortex (vmPFC). ${ }^{23}$

Three-dimensional (3-D) Monte Carlo (MC) models of light transport are commonly used to quantify the photon dosage in brain imaging studies and recently have been used to assess various t-PBM strategies. ${ }^{24}$ The complex interaction between photons and human tissue generally follows the radiative transfer equation (RTE). Although analytical solutions to the RTE exist in simple geometries, they are inherently limited since they assume homogenous or simple layer-dependent tissue properties, with predefined depths for skin, skull, meningeal layers, cerebrospinal fluid (CSF), and brain. This "peel the onion" stratification of tissue properties ignores the irregular shapes of human tissues, which are especially noticeable in the human head. Some simplified versions of the RTE can be applied to arbitrary shapes, however, they also have limitations; for instance, the diffusion equation assumes highly scattering media, and therefore, is not well-suited for photon simulations in the head due to the presence of low-scattering CSF. Given the difficulties of conventional light-transport solutions, we used an in-house developed voxel-based Monte Carlo approach $(\mathrm{MCX})^{25,26}$ for photon migration, accelerated by graphic processing units (GPU).

MCX is capable of modeling light transport in arbitrarily complex 3-D anatomy using a rasterized 3-D heterogeneous domain. Each voxel is associated with a specific tissue type (skin, skull, meningeal layers, CSF, or brain), derived from a segmented anatomical scan, such as those obtained from MRI. In an MCX simulation, a large number of photon packets are launched and their random interactions with the tissues are simulated and recorded in parallel. Every simulated photon packet is first launched from the source, then traverses through the heterogeneous domain following a series of random scattering events determined by known probability distributions. For every voxel along a photon's trajectory, the energy of the photon packet attenuates according to the Beer-Lambert law, and such energy loss is accumulated, ${ }^{25,27}$ resulting in the volumetric energy deposition and fluence. The MC method is known to be highly accurate and often used as the "gold-standard" solution. The GPU-acceleration allows to interrogate various positions of illumination and wavelength settings effectively.

The remainder of this paper is organized as follows. In Sec. 2, the utilized brain atlas model and the tissues optical properties in the MC simulations are described. In addition, we also provide rationales for and details of the types and locations of light sources targeting the vmPFC and dIPFC regions. In Sec. 3, for each transcranial and intranasal source location, we access the respective dosimetry at different cortical regions of the brain atlas and for various wavelengths. Finally, we discuss how the simulation results could be used to guide the clinical dosage in t-PBM treatments and the trade-offs between different source locations for treating the regions of interest in Sec. 4.

\section{Materials and Methods}

In this section, we first discuss the brain atlas anatomical model used in our MC simulations, particularly the optical properties of each brain tissue region. This is followed by our rationale on the selection of the position and form of each light source. Finally, we discuss our simulation settings and the output quantities used to assess the light deposition.

\subsection{Head Models and Tissue Optical Properties}

The Colin27 atlas ${ }^{28,29}$ was used for all simulations in this study. While there are 12 tissue types in the original Colin27 atlas, for simplicity, we combined similar tissues together and created a simplified atlas model containing eight different brain tissue 
types: white matter (WM), gray matter (GM), CSF, skull, muscles, skin/muscles, fat, and blood vessels. The dura and marrow tissues were mapped to the skull due to the lack of distinctive optical tissue properties for these two tissue types. The dimensions of the Colin 27 volume are $362 \times 434 \times 362$ voxels with a voxel size of $0.5 \times 0.5 \times 0.5 \mathrm{~mm}^{3}$. The air pockets in the atlas head were modeled using optical properties of air.

The absorbance of light inside a tissue is generally characterized by the absorption coefficient $\mu_{a},(1 / \mathrm{mm})$ according to the Beer-Lambert law, ${ }^{30}$ while the chance of scattering of these photons is described by the scattering coefficient $\mu_{s}$. In addition, the anisotropy factor $g$ describes the directionality of a scattering event - a value of 0 indicates isotropic scattering and a value close to 1 indicates forward-direction dominant scattering. The $g$ value for brain tissue has a typical value of $0.9 .^{31}$ The combined effect of $\mu_{s}$ and $g$ is measured by the reduced scattering coefficient, defined by $\mu_{s}^{\prime}=\mu_{s}(1-g)$. The higher values of $\mu_{s}$ and $\mu_{s}^{\prime}$ indicate higher scattering counts per unit travel length of the photon. The refractive indices $n$ of all brain tissues are assumed to be 1.37 .

In this study, we simulated light deposition at five wavelengths commonly used in NIR applications-670, 810, 850,
980, and $1064 \mathrm{~nm}$. These wavelengths have been widely used in published studies, many of which correspond well with minima in the absorption spectra of different tissues within the human body. ${ }^{32}$ Although the optical properties of brain tissues have been reported previously, determining the best values for these eight selected tissue types is nontrivial, primarily due to the fact that many of these studies were conducted using different measurement techniques, instrumentation, and population age groups. All the optical properties used in our stimulation study are summarized in Table 1.

Specifically, the values of optical properties were taken from the literature, including muscles, ${ }^{33,34}$ blood vessels (based on circulating blood values), ${ }^{35}$ skull, ${ }^{36,37}$ skin, ${ }^{38} \mathrm{CSF},{ }^{39}$ and GM/ WM. ${ }^{40}$ Since the optical properties for CSF were not directly available for 850 and $980 \mathrm{~nm},{ }^{39}$ the reduced scattering coefficient $\mu_{s}^{\prime}$ for CSF at these two wavelengths was assumed to be $0.01 \mathrm{~mm}^{-1}$, whereas the absorption coefficient of CSF was estimated to be close to that of water. ${ }^{41}$ In fact, the known absorption coefficients of CSF for 670 and $810 \mathrm{~nm}^{39}$ closely mimic the absorption coefficients of water at 850 and $980 \mathrm{~nm}$, i.e., 0.000475 and $0.00219 \mathrm{~mm}^{-1}$, respectively, according to Ref. 41 , or similarly, 0.000412 and $0.0023 \mathrm{~mm}^{-1}$

Table 1 Optical parameters of brain tissues at $670,810,850,980$, and $1064 \mathrm{~nm}$. $\mu_{s}$, scattering coefficient (1/mm); $g$, anisotropy factor; $\mu_{s}^{\prime}$, reduced scattering coefficient $(1 / \mathrm{mm})$; and $\mu_{a}$, absorption coefficient $(1 / \mathrm{mm})$. All $g$ values marked with "—" are set to $0.89 ; \mu_{s}$ (or $\left.\mu_{s}^{\prime}\right)$ marked with "-" are calculated by $\mu_{s}^{\prime}$ (or $\mu_{s}$ ) and $g$.

\begin{tabular}{|c|c|c|c|c|c|c|c|c|c|c|c|c|}
\hline \multirow[b]{2}{*}{ Tissues } & \multicolumn{4}{|c|}{$670 \mathrm{~nm}$} & \multicolumn{4}{|c|}{$810 \mathrm{~nm}$} & \multicolumn{4}{|c|}{$850 \mathrm{~nm}$} \\
\hline & $\mu_{s}$ & $\mu_{s}^{\prime}$ & $\mu_{a}$ & $g$ & $\mu_{s}$ & $\mu_{s}^{\prime}$ & $\mu_{a}$ & $g$ & $\mu_{s}$ & $\mu_{s}^{\prime}$ & $\mu_{a}$ & $g$ \\
\hline WM & 40.1 & - & 0.07 & 0.85 & 38 & - & 0.092 & 0.87 & 35 & - & 0.10 & 0.87 \\
\hline GM & 8.4 & - & 0.02 & 0.9 & 7.3 & - & 0.028 & 0.89 & 7 & - & 0.033 & 0.9 \\
\hline CSF & - & 0.01 & 0.0004 & - & - & 0.01 & 0.0026 & - & - & 0.01 & 0.0420 & - \\
\hline Skull & - & 1.19 & 0.0208 & - & - & 1.92 & 0.011 & - & - & 1.87 & 0.011 & - \\
\hline Vessels & 31.5 & - & 0.13 & 0.989 & 28 & - & 0.11 & 0.990 & 27.5 & - & 0.15 & 0.99 \\
\hline Fat & - & 1.21 & 0.00045 & - & - & 1.09 & 0.00054 & - & - & 1.064 & 0.00071 & - \\
\hline Muscle & 7.65 & - & 0.054 & 0.93 & - & 0.704 & 0.028 & - & - & 0.667 & 0.03 & - \\
\hline \multirow[t]{2}{*}{ Skin } & - & 2.42 & 0.056 & - & - & 2.18 & 0.045 & - & - & 2.043 & 0.04 & - \\
\hline & \multicolumn{4}{|c|}{$980 \mathrm{~nm}$} & \multicolumn{4}{|c|}{$1064 \mathrm{~nm}$} & & & & \\
\hline Tissues & $\mu_{s}$ & $\mu_{s}^{\prime}$ & $\mu_{a}$ & $g$ & $\mu_{s}$ & $\mu_{s}^{\prime}$ & $\mu_{a}$ & $g$ & & & & \\
\hline WM & 31 & - & 0.11 & 0.88 & 30 & - & 0.105 & 0.88 & & & & \\
\hline GM & 6 & - & 0.052 & 0.91 & 5.9 & - & 0.053 & 0.91 & & & & \\
\hline CSF & - & 0.01 & 0.048 & - & - & 0.01 & 0.0144 & - & & & & \\
\hline Skull & - & 1.73 & 0.022 & - & - & 1.61 & 0.019 & - & & & & \\
\hline Vessels & 22 & - & 0.2 & 0.987 & 20 & - & 0.13 & 0.985 & & & & \\
\hline Fat & - & 0.99 & 0.0014 & - & - & 0.945 & 0.0054 & - & & & & \\
\hline Muscle & - & 0.58 & 0.049 & - & - & $\sim 0.55$ & $\sim 0.056$ & - & & & & \\
\hline Skin & - & 1.85 & 0.028 & - & - & 2.03 & 0.017 & - & & & & \\
\hline
\end{tabular}


according to Ref. 42. Thus the absorption of water was used for the remaining wavelengths for CSF. The fat absorption coefficients were obtained from Ref. 43 and the reduced scattering coefficients were calculated from those of the adipose tissues. $^{44,45}$

\subsection{Light Source Positions and Regions of Interest}

A triangular surface mesh of the head model was generated using Iso2Mesh, ${ }^{46}$ to locate the desired light source placement on the head, based on the 10-20 system, ${ }^{47}$ and to locate the cortical regions-of-interest. In our experiment, various extracranial positions for the light source were located on the forehead to target the prefrontal cortex (PFC). The extracranial F3 and F4 frontal positions were chosen based on the favorable results obtained in prior studies of photobiomodulation for mood disorders. ${ }^{17}$ The extracranial frontal positions going across the Fp1, Fp2, and FpZ electroencephalography points were also explored based on preliminary results in a case-report of long-term photobiomodulation for MDD. ${ }^{48}$

In addition, three of the tested illumination positions were located inside the nasal cavity, as illustrated in Fig. 1, to test an alternative strategy to treat the PFC: one in the nostril $(\mathrm{N} 1$, red), the second in the mid-nose (N2, green), and the third near the nose ceiling (cribriform plate, N3, blue). The N1 position is easily accessible and marketed devices already exist to allow clipping a red or NIR light source into the nostril. ${ }^{48}$ Accessing the N2 position (mid-nose) would require common nonsurgical ear, nose, and throat (ENT) procedures such as rhinoscopy (nasal endoscopy). The N3 position, i.e., near the cribriform plate, is still speculative due to limited accessibility, but has shown promise for light delivery to the deep brain previous modeling. ${ }^{24}$ For all three nasal-cavity locations, it was assumed that the light source was pointing directly upward. A narrow-beam LED (Model VSMY2850G, Vishay Semiconductors) was modeled using an angular Gaussian beam, by varying the launch direction of the photons with a random zenith angle following a Gaussian distribution with a variance of $0.105 \mathrm{rad}$. The variance was determined based on fitting the emission angular profile of the LED based on its specification.

The extracranial photobiomodulation illumination strategy on the frontal sites (F3-F4 and Fp1-Fp2-FpZ) was assessed by simulating a $4 \times 7$ array of LED lights, simulating the commercially available PhotoMedex Omnilux New-U light source, recently used in a clinical trial for MDD,${ }^{17}$ with the configuration illustrated in Fig. 1(b). This LED array has a uniform 11-mm LED spacing along the long edge and 8-mm space along the short edge. Four transcranial illumination protocols using the Omnilux New-U light source were studied: (1) two sources simultaneously used with one centered at F3 and the other centered at F4, (2) one source centered at F3, (3) one source centered at F4, and (4) one source centered at the FpZ with lateral coverage between Fp1 and Fp2. In all simulations, the source plane aligns tangent to the skin. The illumination pattern of each LED was assumed to follow that of a pencil beam.

For all extracranial and intranasal positions, the target regions-of-interest in the brain were the dIPFC and vmPFC, on the grounds that both are easily accessible and have been implicated in emotion regulation. ${ }^{23}$ We anticipated that the light source placement on F3 and F4 would preferentially illuminate the dlPFC (Brodmann's areas 9 and 46), and that the intranasal placements would instead preferentially target vmPFC. Source positions are illustrated in Fig. 1(b). In addition, we also show locations and names of all cortical ROIs used in our dosage assessments. For each test scenario, $10^{8}$ photons were simulated on an NVIDIA GTX 1080 graphic card using $\mathrm{MCX}^{25}$

\subsection{Photon Dosimetry Assessment and Data Processing}

For each selected wavelength and location configuration of the light source, the normalized energy deposition $(E)$ profiles were calculated using MCX. The values stored in $E$ (unit: $\mathrm{J} / \mathrm{cm}^{3}$ )

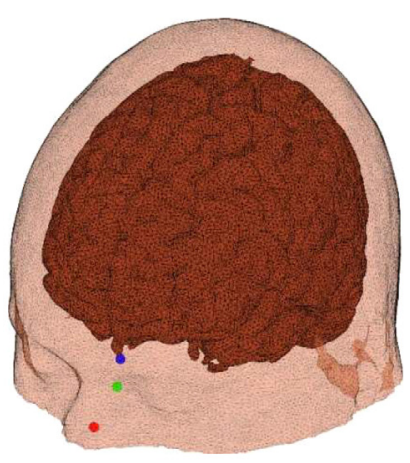

(a)

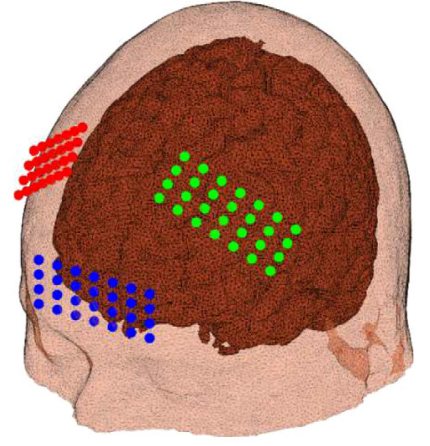

(b)

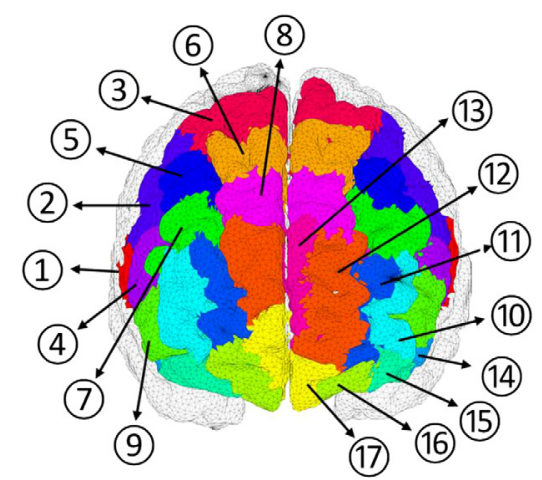

(c)

Fig. 1 Light source configurations: (a) intranasal positions including the nostril (red), mid-nose (green), and near cribriform plate (blue) positions; and (b) transcranial positions using the PhotoMedex Omnilux New-U source (red, F4; green, F3; and blue, Fp1-FpZ-Fp2). In (c), we plot the cortical parcellation map of the Colin 27 atlas, showing the major regions-of-interests including: (1) ventral MC, (2) dorsolateral MC, (3) dorsomedial MC, (4) rostral ventral PMC, (5) dorsolateral PMC, (6) dorsomedial PMC, (7) caudal dorsolateral PFC, (8) caudal dorsomedial PFC, (9) rostral ventrolateral PFC, (10) rostral dorsolateral inferior PFC, (11) rostral dorsolateral superior PFC, (12) rostral dorsal PFC, (13) rostral medial PFC, (14) ventrolateral orbito PFC, (15) ventral orbito FC, (16) ventromedial orbito FC, and (17) ventromedial PFC (MC, motor cortex; PMC, premotor cortex; PFC, prefrontal cortex; and FC, frontal cortex). 

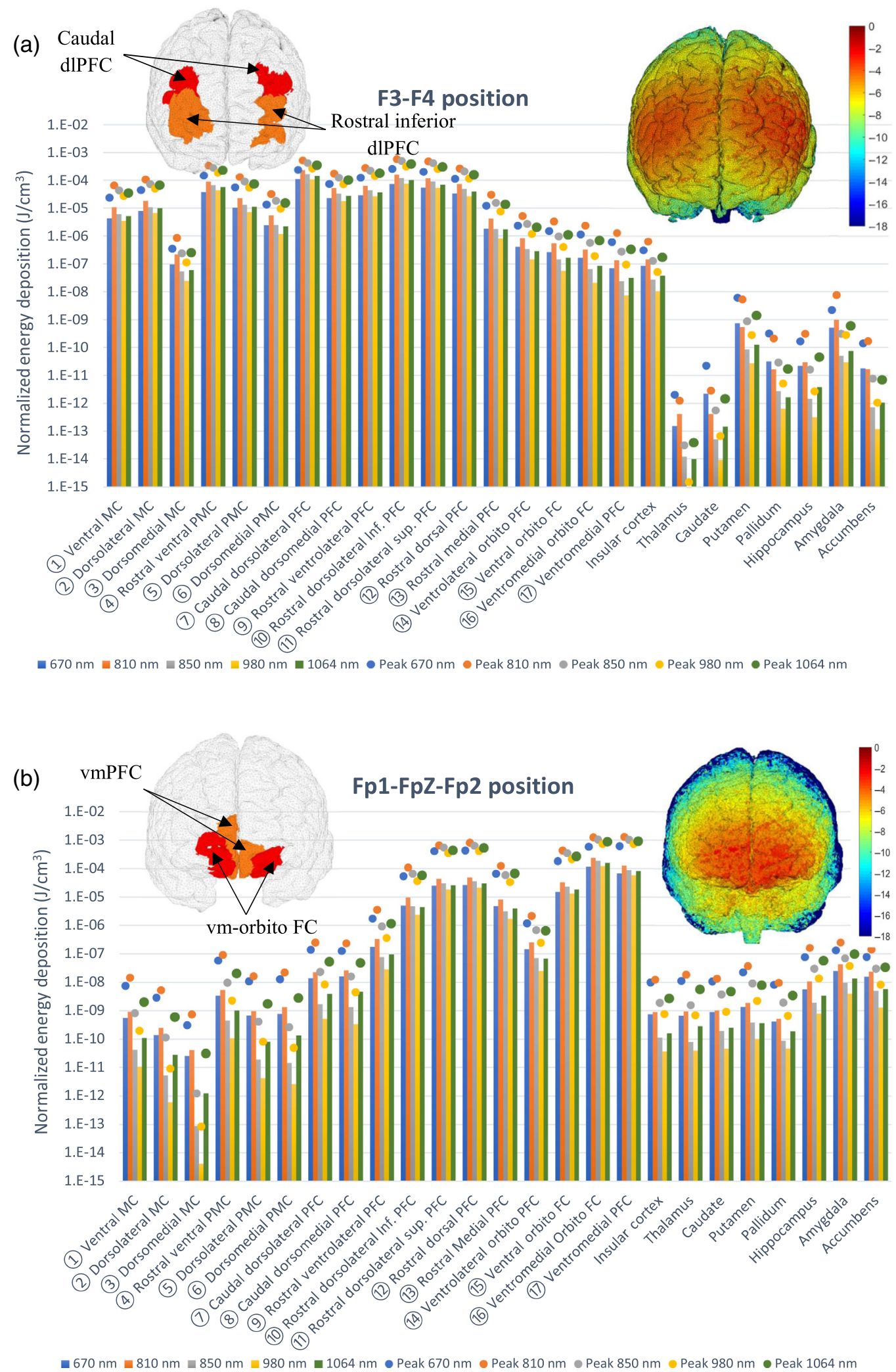

Fig. 2 The average (bars) and peak (dots) energy deposition $\left(\mathrm{J} / \mathrm{cm}^{3}\right)$ after positioning the extracranial light source on the: (a) F3 site and F4 site (two devices simultaneously) and (b) Fp1-FpZ-Fp2 sites (one device). The left inset shows the ROIs that receiving the highest (red) and second highest (orange) energy deposition; the right-inset shows the energy deposition $\left(\mathrm{J} / \mathrm{cm}^{3}\right)$ map on the cortical surface. The locations of the numbered ROls are shown in Fig. 1(c). 

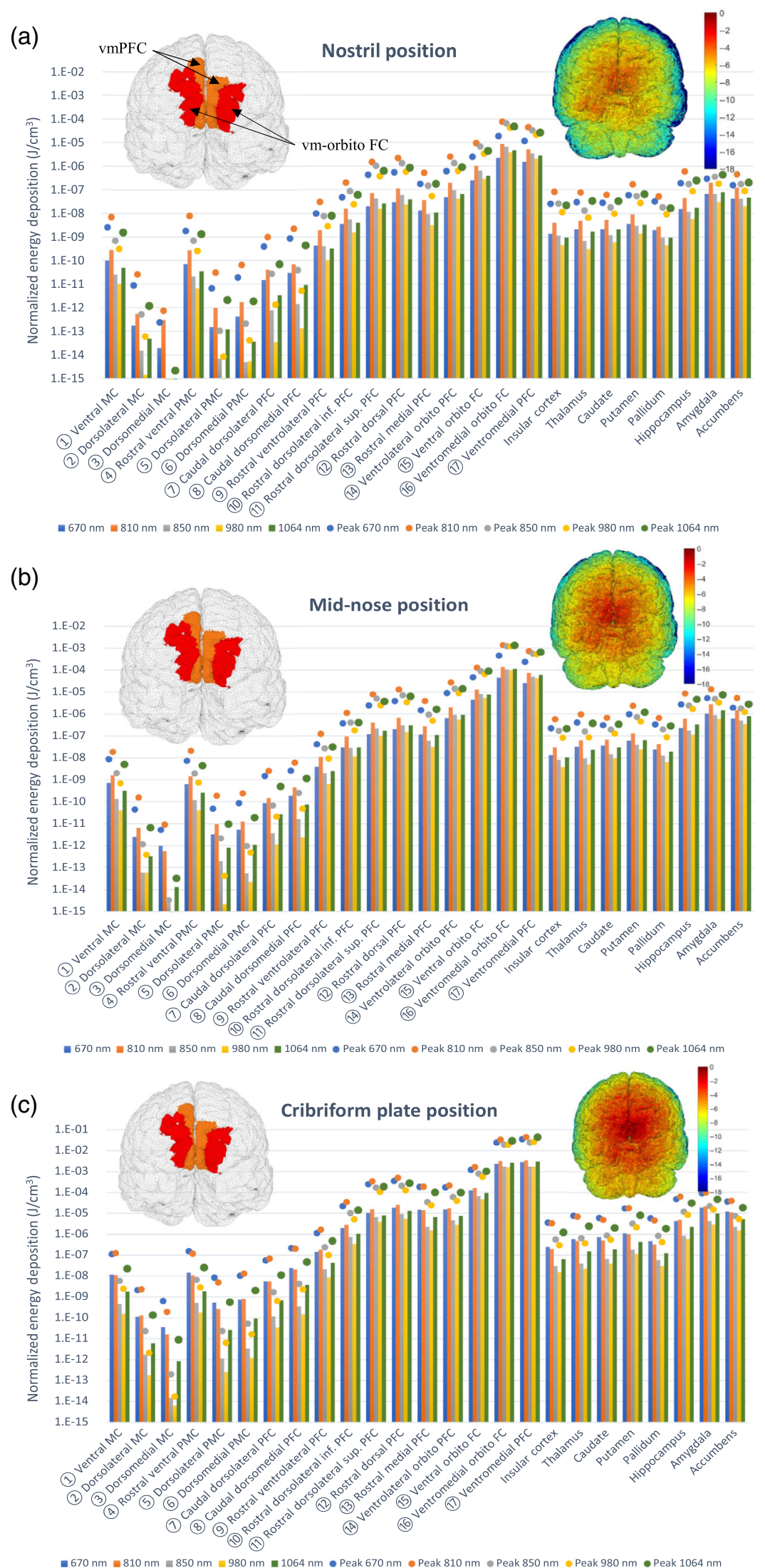

Fig. 3 The average (bars) and peak (dots) energy deposition $\left(\mathrm{J} / \mathrm{cm}^{3}\right)$ after positioning the intranasal light source in the: (a) nostril, (b) mid-nose, and (c) close to the nose ceiling (in proximity of the cribriform plate). The left inset shows the ROls that receiving the highest (red) and second highest (orange) energy deposition; the right-inset shows the energy deposition $\left(\mathrm{J} / \mathrm{cm}^{3}\right)$ map on the cortical surface. The locations of the numbered ROls are shown in Fig. 1(c). 
represent the fractional energy absorbed per unit of tissue volumes. For each unit of energy emitted at the source, the fractional energy loss due to absorbance was calculated by the MC simulation and stored within each $0.5 \times 0.5 \times 0.5 \mathrm{~mm}^{3}$ voxel of the Colin27 atlas. The average absorbed energy $\left(\mathrm{J} / \mathrm{cm}^{3}\right)$ of a particular cortical or subcortical structure was calculated by summing $E$ values along all voxels belonging to each region and then divided by total voxel count of a given structure. In addition, we also calculated the peak energy deposition per voxel by the same structure. The peak absorbance was derived by selecting the 99th percentile of voxels for a given anatomical structures based on the $E$ value of each voxel in the given region. Using a percentile approach leads to reduced variations due to the stochastic nature of MC simulations. Both cortical regions (prefrontal, frontal, and motor cortex) and subcortical regions were considered in this work. To delimitate different subcortical regions, we used a publicly available parcellation and segmentation of subcortical structures - the MarsAtlas model—applied to the Colin27 average brain. ${ }^{49}$ An illustration as well as access to the actual dataset is available online. ${ }^{50}$

In all simulations, the total energy simulated at each source (either point or array) was normalized to be $1 \mathrm{~J}$. An exception was made in the case of the combined F3-F4 illumination, where $1 \mathrm{~J}$ was assumed for each of the F3 and F4 sources (thus a total of $2 \mathrm{~J}$ ), and the deposition values were the combined energy of these two sources. This exception was decided after the model revealed that each of the two sources had negligible deposition of light to the contralateral homologous cortex. The average normalized deposited energy per unit volume can be easily interpreted: for instance, a $1.03 \times 10^{-4} \mathrm{~J} / \mathrm{cm}^{3}$ means that for each joule of energy emitted from the source location $1.03 \times 10^{-4} \mathrm{~J}$ was deposited at the target cortical/subcortical regions in the brain per $\mathrm{cm}^{3}$ of the given tissue. Similarly, the average normalized fluence per unit surface can also be interpreted: for instance, a $1.01 \times 10^{-5} \mathrm{~J} / \mathrm{cm}^{2}$ means that for each joule of energy emitted from the source location $1.01 \times 10^{-5} \mathrm{~J}$ is deposited on the surface of the target brain region per $\mathrm{cm}^{2}$.

\section{Results}

The energy deposition maps from the above simulations are presented in Fig. 2 for the extracranial positions and in Fig. 3 for the intranasal positions. From both plots, it appears that the physical distance between the source location and target, for instance, the cortex, is the dominant factor determining the magnitude of the energy deposition. This is because the light intensity decays exponentially with respect to distance, according to the BeerLambert law. ${ }^{30}$ We note that the average scalp thickness for this model is $6.9 \pm 3.6 \mathrm{~mm}$, whereas the skull thickness is $6.0 \pm$ $1.9 \mathrm{~mm}$ with a detailed breakdown available. ${ }^{51}$ Regarding the extracranial source locations, placing the LED array at the F3 and F4 locations permits preferential illumination of the premotor, motor, and prefontal cortex, on the left and right hemisphere, respectively. The average energy deposition was calculated as $2.34 \times 10^{-4} \mathrm{~J} / \mathrm{cm}^{3}$ for the caudal dlPFC and was consistently above $\sim 10^{-6} \mathrm{~J} / \mathrm{cm}^{3}$ at $810 \mathrm{~nm}$ over the targeted regions with the exceptions of the dorsomedial motor cortex and ventrolateral orbito FC regions (as shown in Fig. 2) that are located further away from the source locations. When comparing the energy depositions from the F3 position onto the brain regions of the left hemisphere with the F4 position onto the right hemisphere regions, the values were found to be comparable with the left hemisphere receiving only about $1.6 \%$ more energy. Both sources, on F3 and F4, had negligible deposition of light to the contralateral, homologous cortex. The Fp1-FpZFp2 location preferentially delivered light to the most anterior portion of the prefontal and frontal cortex regions, corresponding to the Brodmann areas 10 and 11 with average values above $\sim 10^{-6} \mathrm{~J} / \mathrm{cm}^{3}$. The highest average energy deposition was observed at the ventromedial orbito FC and ventromedial PFC regions (shown in Fig. 3). The vmPFC region, known to be related to emotional regulation, was noted to have strong deposition at this position with a simulated average value of $1.27 \times$ $10^{-4} \mathrm{~J} / \mathrm{cm}^{3}$ at $810 \mathrm{~nm}$, whereas the ventromedial orbito $\mathrm{FC}$ showed an average deposition of $2.41 \times 10^{-4} \mathrm{~J} / \mathrm{cm}^{3}$ at $810 \mathrm{~nm}$.

Among the intranasal positions, the proximity of the cribriform plate to the vmPFC cortex, as well as other neighboring cortical regions, led to a very high energy deposition, with average values of up to $0.0034 \mathrm{~J} / \mathrm{cm}^{3}$ per joule of energy emitted from the source at $810 \mathrm{~nm}$. In comparison, this average energy deposition on vmPFC was 46-fold stronger than illuminating at the mid-nose position $\left(7.41 \times 10^{-5} \mathrm{~J} / \mathrm{cm}^{3}\right.$ at $\left.810 \mathrm{~nm}\right)$ and 658 -fold more than the nostril position $\left(5.15 \times 10^{-6} \mathrm{~J} / \mathrm{cm}^{3}\right.$ at $810 \mathrm{~nm})$. These differences among intranasal light sources are depicted visually in Fig. 4.

From the simulations shown in Figs. 2 and 3, besides the position of the light source, the wavelength also appears to play an important role in determining the magnitude of the energy deposition. In general, for the five wavelengths explored, there was a clear trend showing that $810 \mathrm{~nm}$ offered the highest light deposition onto the brain, followed closely by 1064 and

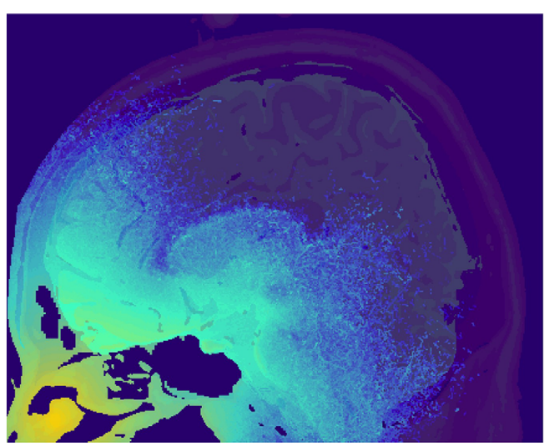

(a)

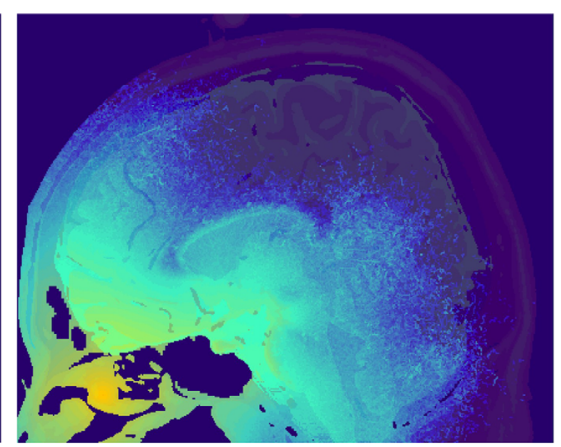

(b)

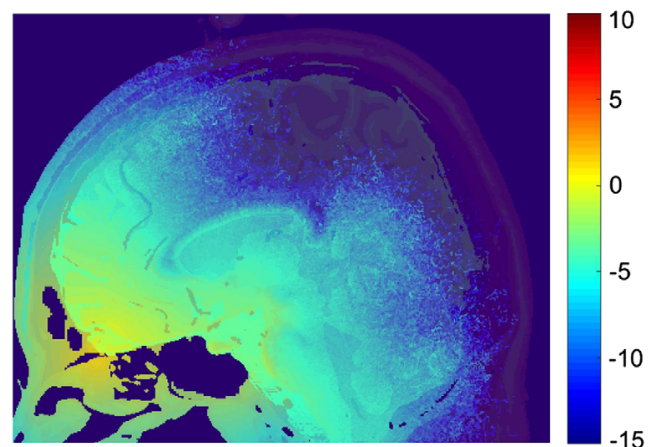

(c)

Fig. 4 Sagittal section plots of the normalized energy deposition (in log-scale) results for (a) the nostril illumination, (b) the mid-nose illumination, and (c) the cribriform plate illumination. All results are simulated with the optical properties at $810 \mathrm{~nm}$. 


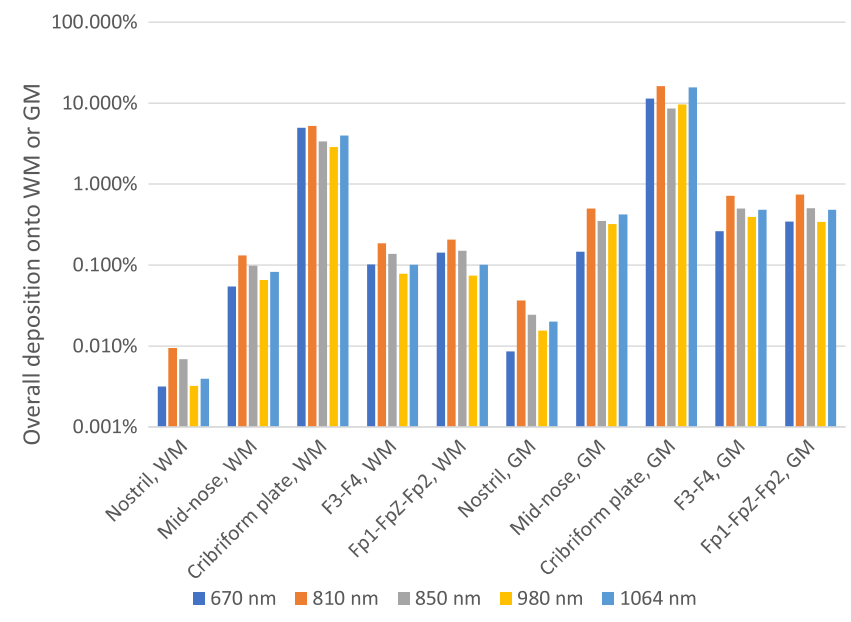

Fig. 5 Overall energy deposition onto the gray and white matters based on different source locations.

$850 \mathrm{~nm}$. The 850-nm wavelength typically deposited less energy than $810 \mathrm{~nm}$ to the brain targets due to the higher absorption by CSF of the 850-nm light; however, when there is little intervening CSF between the source location and the target region, 850 and $810 \mathrm{~nm}$ perform similarly. The 1064-nm light usually offered better deposition onto the brain than 670,850 , and $980 \mathrm{~nm}$. These trends are illustrated in Fig. 5. The 670-nm exhibits a low deposition onto the brain with the exception of the cribriform plate source location. However, Figs. 2 and 3 show similar deposition from the $670-\mathrm{nm}$ and 810 -nm wavelengths when looking at the brain regions located further away from the source.

Analyzing the peak (99th percentile of voxels) and average energy deposition values across different brain regions provides similar conclusions concerning the deposition patterns onto the brain, that is, an absolute difference of about one-to-two orders of magnitude is observed on average between these two indicators from the results shown in Figs. 2 and 3, mainly due to the spatial distance variability with respect to the source location between voxels within a given structure, whether subcortical or cortical. At a cortical level, the variability in distance from the light source is likely explained by the spreading of functional-anatomical regions over alternating folding structures, which are part of the cortical gyri. The overall depositions onto the GM and WM tissues at $810 \mathrm{~nm}$, denoting the percentage deposited of the total energy emitted by the light source, are summarized in Fig. 5. The nostril provides an almost negligible deposition onto the brain structures, whereas the cribriform plate is simulated to deposit almost $\sim 20 \%$ of the source energy onto the brain (WM and GM). The mid-intranasal, the Fp1-FpZFp2, and F3-F4 locations are shown to have relatively similar brain depositions of about $1 \%$ of the total transmitted energy at $810 \mathrm{~nm}$.

The box plots in Fig. 6 provide a summary of the fluence $\left(\mathrm{J} / \mathrm{cm}^{2}\right)$ at $810 \mathrm{~nm}$ delivered to various brain regions-of-interest that exhibited the highest energy depositions as summarized in Figs. 2 and 3, based on each specific source location. The cribriform plate shows the highest fluence values for both the vm-orbito FC (interquartile values of 0.0072 and $5.19 \times 10^{-4} \mathrm{~J} / \mathrm{cm}^{2}$ ) and vmPFC (interquartile values of 0.0065 and $2.83 \times 10^{-4} \mathrm{~J} / \mathrm{cm}^{2}$ ). While the Fp1-FpZ-Fp2 location provides a better illumination at the vm-orbito FC region, the mid-nose location shows a tighter distribution indicating a more uniform illumination for both the vm-orbito FC and vmPFC regions. The fluence distributions of the $\mathrm{F} 3-\mathrm{F} 4$ source onto the caudal and rostral inferior dIPFC are similar in magnitude to the highest observed fluence values for the Fp1-FpZ-Fp2 source.

\section{Discussion}

Our simulations suggest that selective targeting of dlPFC and of vmPFC with PBM for regulation of emotion is feasible, and that transcranial and intranasal PBM are both possible routes of light delivery. The illustrative quantities used in this section are assumed to be average deposition values unless stated otherwise. The transcranial position of the light source on F3-F4 led to preferential delivery of the light onto the dlPFC, when compared to other regions of the PFC. The light reached the dlPFC with an average magnitude of $10^{-4} \mathrm{~J} / \mathrm{cm}^{3}$ per joule delivered. Based on Fig. 2, the positioning on F3-F4 allows us to selectively target dIPFC with at least two orders of magnitude higher average energy deposition when compared to vmPFC (range: $10^{-6}$ to $10^{-8} \mathrm{~J} / \mathrm{cm}^{3}$ ). When shedding the light only on either $\mathrm{F} 3$ or $\mathrm{F} 4$, we also found that it is possible to unilaterally and

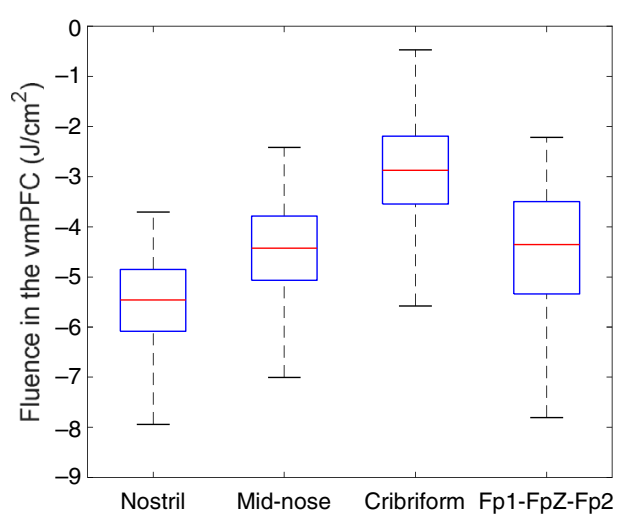

(a)

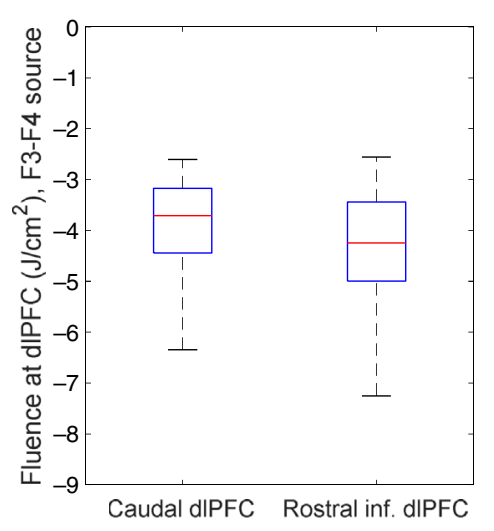

(b)

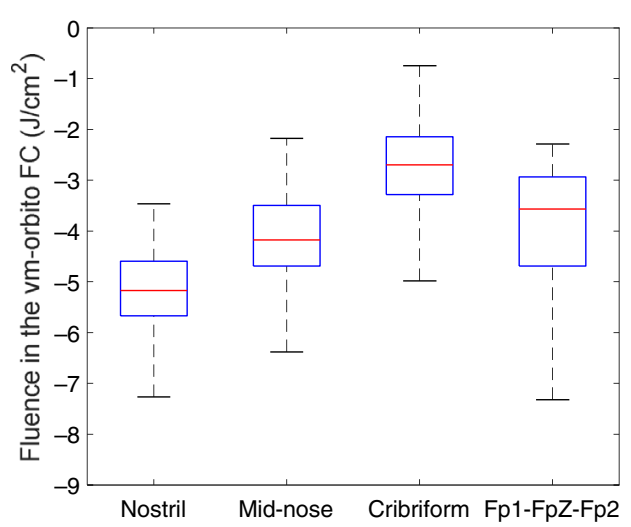

(c)

Fig. 6 Box plots summarizing the fluence $\left(\mathrm{J} / \mathrm{cm}^{2}\right)$ distribution (a) on the vmPFC region and (b) we show the fluence distribution on the caudal dIPFC and on the rostral inferior dIPFC for a source located on F3F4; (c), on the vm-orbitoFC region for sources located intranasally and on Fp1-FpZ-Fp2, as illustrated in Fig. 1. All the values are for $810 \mathrm{~nm}$ and the $y$-scale is on a log-10 scale. 
selectively irradiate left and right dIPFC. In fact, in accordance with the exponential decay of the light, and due to the large interhemispheric distance, the magnitude of light on the dlPFC at the nontargeted hemisphere was negligible $\left(<10^{-14} \mathrm{~J} / \mathrm{cm}^{3}\right)$ when compared to the targeted dIPFC. Identical and specular results were obtained when F3 and F4 were chosen to target left and right dlPFC, respectively. The transcranial position of the light on Fp1-FpZ-Fp2 led to preferential light delivery to the vm-orbitoFC and vmPFC $\left(\sim 10^{-4} \mathrm{~J} / \mathrm{cm}^{3}\right)$. Interestingly, this position was only marginally selective for vmPFC, since there was less than one order of magnitude difference between the energy deposition calculated on the vmPFC and the rostral dlPFC $\left(1.27 \times 10^{-4}\right.$ and $4.89 \times 10^{-5} \mathrm{~J} / \mathrm{cm}^{3}$, respectively, at $810 \mathrm{~nm})$.

The intranasal positioning of the light source led to preferential shedding of the light on the vm-orbitoFC and vmPFC, with at least two orders of magnitude difference in energy deposition compared to the dlPFC, and one order of magnitude difference from any other brain region. As expected, the deposition onto the cortex increased exponentially as the light source was moved nearer to the target area. The source positioned in the nostril suffered from large attenuation, with the light reaching the brain at the peak region of the vmPFC at the order of $10^{-5} \mathrm{~J} / \mathrm{cm}^{3}$. When the source was positioned in the mid-nose region, the attenuation was the same as the average attenuation for transcranial delivery, on the order of $10^{-4} \mathrm{~J} / \mathrm{cm}^{3}$. The cribriform plate positioning of the light source showed the least attenuation $\left(10^{-3} \mathrm{~J} / \mathrm{cm}^{3}\right)$, which led to the highest average energy deposition on the cortex compared to all the other light source positions, both intranasal and transcranial, examined in this paper.

These results suggest limited utility of the nostril positioning of the light source for direct PBM of the cortex. On the contrary, the mid-nose position provided comparable light delivery with respect to the transcranial positions and reached equivalent energy deposition to vmPFC and vm-orbitoFC when compared to transcranial light sources positioned on Fp1-FpZ-Fp2. The mid-nose was, however, more selective as it only marginally irradiated the dlPFC (mid-nose: $10^{-7} \mathrm{~J} / \mathrm{cm}^{3}$ versus Fp1FpZ-Fp2: $10^{-5} \mathrm{~J} / \mathrm{cm}^{3}$ ). The mid-nose position might offer an advantage over the transcranial position of the light source in case of brain atrophy, when irradiating vmPFC and vmorbitoFC. However, the mid-nose position might cause patient discomfort and it would likely require delivery by specialized operators and, even then, it might be associated with risk of nose bleeding. Patients with mood or anxiety disorders who do not respond to transcranial applications on vmPFC might benefit from higher energy depositions obtained from the convergence of transcranial and mid-nose light delivery. The positioning of the light source near the cribriform plate achieves the highest energy deposition onto the vmPFC and vm-orbitoFC regions, however, with available technology, access to this region is challenging based on benefit-risk ratio of related ENT procedures.

The subcortical structures located in the deeper regions of the brain, which are relevant to emotion regulation and to mood and anxiety disorders, such as the thalamus, hippocampus, and amygdala, received negligible light from the nostril source position, but both the mid-nose and the cribriform plate positions could provide a modest, and likely still insufficient amount of light to deeper brain regions $\left(10^{-5} \mathrm{~J} / \mathrm{cm}^{3}\right.$ or less). The prospects of deep brain PBM appear to be limited at present, at least with the examined transcranial and intranasal routes.
Most importantly, our simulations suggest that current studies on t-PBM use a dose of light delivered to F3-F4 or Fp1-FpZ-Fp2 sufficient to adequately irradiate one quarter to half of the target regions (the upper one or two quartiles of vm-orbitoFC, vmPFC, and caudal dlPFC in Fig. 6). Results that were obtained in animal models indicate that for $810-\mathrm{nm}$ light; the effective fluence required to be achieved at the target tissue for neuromodulation is between 0.3 and $3 \mathrm{~J} / \mathrm{cm}^{2}$ per session. ${ }^{52}$ Because in our above simulations, the derived quantities were all normalized by assuming the total emitted source energy was $1 \mathrm{~J}$. In order to determine whether our calculated energy depositions are sufficient, we need to scale our outputs using the power density $\left(P_{s}\right.$, in $\left.\mathrm{W} / \mathrm{cm}^{2}\right)$ and illumination duration $(t)$ parameters of an actual PBM source. As a t-PBM treatment parameter, fluence (energy density) at skin level $\left(\Phi_{0}\right.$, in $\left.\mathrm{J} / \mathrm{cm}^{2}\right)$ can be calculated as $\Phi_{0}=P_{s} \times t$.

The fluence value at the target tissue can be estimated by proportionally scaling the fluence at skin level, based on the attenuation values reported in this work (Fig. 6). Let us then consider three prototypical treatment scenarios for a t-PBM $(810 \mathrm{~nm})$ session and the expected fluence at the target level for the two transcranial source locations (F3-F4 and Fp1FpZ-Fp2). The treatment window is assumed to be $28.4 \mathrm{~cm}^{2}$ as described in Sec. $2(4 \mathrm{~cm} \times 7.1 \mathrm{~cm})$. There are effectively two treatment windows for the F3-F4 source locations (bilateral treatment), but the simulated total energy was $2 \mathrm{~J}$ instead of $1 \mathrm{~J}$ used for the other single source location, leading to similar calculations for the therapeutic fluence. Based on the above results, we devised four sample PBM treatment scenarios. The therapeutic fluence at various brain targets in each case is summarized in Table 2.

It is expected that wavelengths other than $810 \mathrm{~nm}$ would sufficiently irradiate an even smaller area of the target brain tissue, due to lower penetration of the light. However, both average and peak energy depositions at the primary targets are strikingly similar for the different wavelengths (670 to $1064 \mathrm{~nm})$ and remain within the same order of magnitude. Treatment scenario-I seems preferable, whereas scenario-III would irradiate sufficiently only one quarter of the target tissue. Presumably, even for scenario-III, multiple weekly sessions might lead to a cumulative light exposure sufficient to neuromodulate a larger proportion of the target tissues than what estimated for a single session. This speculation is consistent with the clinical and preclinical observations supporting that multiple sessions are more effective than a single session in determining psychological and behavioral improvements. Alternatively, irradiating a larger surface of the forehead might also translate into neuromodulation of a larger area of the (most superficial) target tissues. In comparison, scenario-IV shows the inefficacy of the nostril positions to reach those targets.

The limitations of our work are mostly related to the use of modeling, as opposed to in vivo dosimetry in primates, and to the assumptions made. For instance, our model does not address the heterogeneity of optical properties within tissues, since each tissue is assumed to have homogeneous, average properties. Furthermore, we averaged the properties of different tissues such as skin and muscle and the properties of bone and marrow. In this study, we mainly focused on the relative/normalized energy deposition for each illumination strategy and wavelength. To predict practical PBM device performance, one must scale our normalized results by the power of the actual illumination source, which should satisfy the safety requirements 
Table 2 Therapeutic fluence at brain targets from various source locations for four treatment scenarios at $810 \mathrm{~nm}$.

\begin{tabular}{|c|c|c|c|c|c|}
\hline \multirow[b]{2}{*}{ Scenario } & \multirow[b]{2}{*}{$\begin{array}{l}\text { Skin irradiance - time of exposure } \\
\text { (total delivered energy) }\end{array}$} & \multirow[b]{2}{*}{$\begin{array}{c}\text { Metrics (peak } \\
\text { average of } \\
\text { the upper quantile) }\end{array}$} & \multicolumn{3}{|c|}{ Therapeutic fluence at brain targets (source location) } \\
\hline & & & $\begin{array}{c}\text { vm-orbitoFC } \\
\text { (Fp1-FpZ-Fp2) } \\
\left(\mathrm{J} / \mathrm{cm}^{2}\right)\end{array}$ & $\begin{array}{c}\text { vmPFC } \\
\text { (Fp1-FpZ-Fp2) } \\
\left(\mathrm{J} / \mathrm{cm}^{2}\right)\end{array}$ & $\begin{array}{l}\text { dIPFC } \\
(\mathrm{F} 3-\mathrm{F} 4) \\
\left(\mathrm{J} / \mathrm{cm}^{2}\right)\end{array}$ \\
\hline \multirow[t]{2}{*}{1} & $300 \mathrm{~mW} / \mathrm{cm}^{2}-5 \mathrm{~min}(2.556 \mathrm{~kJ})$ & Peak & 3.00 & 0.82 & 1.72 \\
\hline & & Median & 0.69 & 0.11 & 0.50 \\
\hline \multirow[t]{2}{*}{ II } & $30 \mathrm{~mW} / \mathrm{cm}^{2}-30 \mathrm{~min}(1.530 \mathrm{~kJ})$ & Peak & 1.80 & 0.49 & 1.03 \\
\hline & & Median & 0.41 & 0.07 & 0.30 \\
\hline \multirow[t]{3}{*}{ III } & $15 \mathrm{~mW} / \mathrm{cm}^{2}-40 \mathrm{~min}(1.022 \mathrm{~kJ})$ & Peak & 1.20 & 0.32 & 0.69 \\
\hline & & Median & 0.27 & 0.05 & 0.20 \\
\hline & & & vm-orbitoFC (nostril) & vmPFC (nostril) & \\
\hline \multirow[t]{2}{*}{ IV } & $(1 \mathrm{~kJ})$ & Peak & 0.025 & 0.014 & \\
\hline & & Median & 0.007 & 0.003 & \\
\hline
\end{tabular}

specified by the ANSI standard Z136.1 (2014). According to this standard, the maximum permissible exposure (MPE) of the light source is organ (skin versus retina) and wavelength dependent. For skin exposure, the MPE at $670 \mathrm{~nm}$ is around $200 \mathrm{~mW} / \mathrm{cm}^{2}$; the MPE increases to $400 \mathrm{~mW} / \mathrm{cm}^{2}$ at $850 \mathrm{~nm}$. Once the illumination area and wavelength are determined, the maximum source power can be calculated. One can also limit the source power by investigating maximum temperature increase. Unfortunately, rigorously modeling temperature change is quite challenging, requiring solving both light and bioheat transfer problems, as well as considering cooling by blood perfusion and surface air convection (especially for intranasal cases). We will address these challenges in our future studies.

\section{Conclusions}

In this study, we present an MC simulation-based photon dosimetry assessment with a focus on estimating light delivery to the dIPFC and vmPFC regions. Such a study can potentially lead to identification of optimal illumination locations, source types, and wavelengths for effective PBM strategies (or device designs) to treat depression and other disorders, associated with dIPFC and vmPFC dysfunction. Our simulations suggest that it is possible to selectively modulate vmPFC and dlPFC using NIR light delivered transcranially or intranasally. Various illumination positions and wavelengths are simulated using a human brain atlas; measurements of the light deposition in different brain regions are calculated. Our results also suggest that typical t-PBM doses are suboptimal for neuromodulation of the entire surface of target brain regions. Therefore, repeated sessions of $\mathrm{t}-\mathrm{PBM}$ or greater doses per session or larger treatment windows might be needed to achieve favorable clinical outcomes. Finally, intranasal PBM might be a promising alternative to t-PBM to achieve neuromodulation of vmPFC and vm-orbitoFC.

\section{Disclosures}

Dr. P. Cassano's salary was supported by the Harvard Psychiatry Department (Dupont Warren Fellowship and Livingston Award), the Brain and Behavior Research Foundation (NARSAD Young Investigator Award), and a Photothera Inc. unrestricted grant. Drug donation from Teva. Travel reimbursement from Pharmacia \& Upjohn. Dr. Cassano has received consultation fees from Janssen Research and Development. Dr. Cassano has filed several patents related to the use of NIR light in psychiatry. PhotoMedex, Inc. supplied four devices for a clinical study. Dr. Cassano has recently received unrestricted funding from Litecure Inc. to conduct a study on t-PBM for the treatment of MDD and to conduct a study on healthy subjects. He co-founded a company (Niraxx Light Therapeutics) focused on the development of new modalities of treatment based on NIR light; he is a consultant for the same company. He also received funding from Cerebral Sciences to conduct a study on t-PBM for generalized anxiety disorder. Dr. Q. Fang has received consultation fees from Kernel LLC.

\section{Acknowledgments}

This work was supported by the U.S. National Institutes of Health (NIH) under Grant Nos. R01-GM114365, R01CA204443, and R01-EB026998.

\section{References}

1. M. R. Hamblin, "Shining light on the head: photobiomodulation for brain disorders," BBA Clin. 6, 113-124 (2016).

2. F. Salehpour et al., "Brain photobiomodulation therapy: a narrative review," Mol. Neurobiol. 55(8), 6601-6636 (2018).

3. P. Cassano and M. A. Caldieraro, "Photobiomodulation," in The Massachusetts General Hospital Guide to Depression, 1st ed., Springer International Publishing, New York (2018).

4. ClinicalTrials.gov, "Clinical trials database," https://clinicaltrials.gov/ ct2 (26 September 2018).

5. W. Yu et al., "Photomodulation of oxidative metabolism and electron chain enzymes in rat liver mitochondria," Photochem. Photobiol. 66(6), 866-871 (1997).

6. N. Mochizuki-Oda et al., "Effects of near-infra-red laser irradiation on adenosine triphosphate and adenosine diphosphate contents of rat brain tissue," Neurosci. Lett. 323(3), 207-210 (2002).

7. U. Oron et al., "Ga-As $(808 \mathrm{~nm})$ laser irradiation enhances ATP production in human neuronal cells in culture," Photomed. Laser Surg. 25(3), $180-182$ (2007). 
8. M. Yamaura et al., "Low level light effects on inflammatory cytokine production by rheumatoid arthritis synoviocytes," Lasers Surg. Med. 41(4), 282-290 (2009).

9. J. J. Anders, "The potential of light therapy for central nervous system injury and disease," Photomed. Laser Surg. 27(3), 379-380 (2009).

10. J. Khuman et al., "Low-level laser light therapy improves cognitive deficits and inhibits microglial activation after controlled cortical impact in mice," J. Neurotrauma 29(2), 408-417 (2012).

11. T. Ando et al., "Comparison of therapeutic effects between pulsed and continuous wave 810 -nm wavelength laser irradiation for traumatic brain injury in mice," PLoS One 6(10), e26212 (2011).

12. W. Xuan et al., "Low-level laser therapy for traumatic brain injury in mice increases brain derived neurotrophic factor (BDNF) and synaptogenesis," J. Biophotonics 8(6), 502-511 (2015).

13. L. F. De Freitas and M. R. Hamblin, "Proposed mechanisms of photobiomodulation or low-level light therapy," IEEE J. Sel. Top. Quantum Electron. 22(3), 348-364 (2016).

14. F. Schiffer et al., "Psychological benefits 2 and 4 weeks after a single treatment with near infrared light to the forehead: a pilot study of 10 patients with major depression and anxiety," Behav. Brain Funct. 5(1), 46 (2009).

15. P. Cassano et al., "Near-infrared transcranial radiation for major depressive disorder: proof of concept study," Psychiatry J. 2015, 1-8 (2015).

16. S. G. Disner, C. G. Beevers, and F. Gonzalez-Lima, "Transcranial laser stimulation as neuroenhancement for attention bias modification in adults with elevated depression symptoms," Brain Stimul. 9(5), 780-787 (2016).

17. P. Cassano et al., "Transcranial photobiomodulation for the treatment of major depressive disorder: the ELATED-2 Pilot trial," Photomed. Laser Surg. 36(12), 634-646 (2018).

18. M. D. Ho et al., "Increased functional connectivity in default mode network associated with application of transcranial, light-emitting diodes to treat chronic aphasia: case series," Poster presented at the Forty Fourth Ann. Meet. Int. Neuropsychol. Soc., Boston, Massachusetts (2016).

19. T. A. Henderson and L. D. Morries, "Near-infrared photonic energy penetration: can infrared phototherapy effectively reach the human brain?" Neuropsychiatr. Dis. Treat. 11, 2191-2208 (2015).

20. J. R. Jagdeo et al., "Transcranial red and near infrared light transmission in a cadaveric model," PLoS One 7(10), e47460(2012).

21. C. E. Tedford et al., "Quantitative analysis of transcranial and intraparenchymal light penetration in human cadaver brain tissue," Lasers Surg. Med. 47(4), 312-322 (2015).

22. J. T. Hashmi et al., "Effect of pulsing in low-level light therapy," Lasers Surg. Med. 42(6), 450-466 (2010).

23. M. Koenigs and J. Grafman, "The functional neuroanatomy of depression: distinct roles for ventromedial and dorsolateral prefrontal cortex," Behav. Brain Res. 201(2), 239-243 (2009).

24. A. Pitzschke et al., "Red and NIR light dosimetry in the human deep brain," Phys. Med. Biol. 60(7), 2921-2937 (2015).

25. Q. Fang and D. A. Boas, "Monte Carlo simulation of photon migration in 3D turbid media accelerated by graphics processing units," Opt. Express 17(22), 20178-20190 (2009).

26. L. Yu et al., "Scalable and massively parallel Monte Carlo photon transport simulations for heterogeneous computing platforms," J. Biomed. Opt. 23(1), 010504 (2018).

27. L. Wang, S. L. Jacques, and L. Zheng, "MCML-Monte Carlo modeling of light transport in multi-layered tissues," Comput. Meth. Programs Biomed. 47(2), 131-146 (1995).

28. C. J. Holmes et al., "Enhancement of MR images using registration for signal averaging," J. Comput. Assist. Tomogr. 22(2), 324-333 (1998).

29. B. Aubert-Broche, A. C. Evans, and L. Collins, "A new improved version of the realistic digital brain phantom," Neuroimage 32(1), 138-145 (2006).

30. L. Kocsis, P. Herman, and A. Eke, "The modified Beer-Lambert law revisited," Phys. Med. Biol. 51(5), N91-N98 (2006).

31. E. Okada et al., "Influence of anisotropy factor on optical properties measured from time-resolved reflectance of cortical tissue," Trans. Jpn. Soc. Med. Biol. Eng. 52, 117-118 (2014).

32. J. Hui et al., "Bond-selective photoacoustic imaging by converting molecular vibration into acoustic waves," Photoacoustics 4(1), 11-21 (2016).

33. A. M. K. Nilsson, R. Berg, and S. Andersson-Engels, "Measurements of the optical properties of tissue in conjunction with photodynamic therapy," Appl. Opt. 34(21), 4609-4619 (1995).
34. R. Simpson et al., "Near-infrared optical properties of ex-vivo human skin and subcutaneous tissues using reflectance and transmittance measurements," Proc. SPIE 2979, 307-313 (1997).

35. A. Roggan et al., "Optical properties of circulating human blood in the wavelength range 400-2500 nm," J. Biomed. Opt. 4(1), 36-46 (1999).

36. A. N. Bashkatov et al., "Optical properties of human cranial bone in the spectral range from 800 to 2000 nm," Proc. SPIE 6163, 616310 (2006).

37. F. Bevilacqua et al., "In vivo local determination of tissue optical properties: applications to human brain," Appl. Opt. 38(22), 4939-4950 (1999).

38. A. N. Bashkatov et al., "Optical properties of human skin, subcutaneous and mucous tissues in the wavelength range from 400 to $2000 \mathrm{~nm}$," J. Phys. D. Appl. Phys. 38(15), 2543-2555 (2005).

39. G. Strangman, M. A. Franceschini, and D. A. Boas, "Factors affecting the accuracy of near-infrared spectroscopy concentration calculations for focal changes in oxygenation parameters," Neuroimage 18(4), 865-879 (2003).

40. A. N. Yaroslavsky et al., "Optical properties of selected native and coagulated human brain tissues in vitro in the visible and near infrared spectral range," Phys. Med. Biol. 47(12), 2059-2073 (2002).

41. L. Kou, D. Labrie, and P. Chylek, "Refractive indices of water and ice in the 065- to 25- $\mu$ m spectral range," Appl. Opt. 32(19), 3531 (1993).

42. H. Buiteveld, J. H. M. Hakvoort, and M. Donze, "Optical properties of pure water," Proc. SPIE 2258, 174-183 (1994).

43. R. L. P. van Veen et al., "Determination of visible near-IR absorption coefficients of mammalian fat using time and spatially resolved diffuse reflectance and transmission spectroscopy," J. Biomed. Opt. 10(5), 054004 (2005).

44. G. Alexandrakis, F. R. Rannou, and A. F. Chatziioannou, “Tomographic bioluminescence imaging by use of a combined optical-PET (OPET) system: a computer simulation feasibility study," Phys. Med. Biol. 50(17), 4225-4241 (2005).

45. S. L. Jacques, "Optical properties of biological tissues: a review," Phys. Med. Biol. 58, R37 (2013).

46. Q. Fang and D. A. Boas, "Tetrahedral mesh generation from volumetric binary and gray-scale images," in IEEE Int. Symp. Biomed. Imaging: From Nano to Macro, pp. 1142-1145 (2009).

47. V. Jurcak, D. Tsuzuki, and I. Dan, "10/20, 10/10, and 10/5 systems revisited: their validity as relative head-surface-based positioning systems," Neuroimage 34(4), 1600-1611 (2007).

48. M. A. Caldieraro et al., "Long-term near-infrared photobiomodulation for anxious depression complicated by Takotsubo cardiomyopathy," J. Clin. Psychopharmacol. 38(3), 268-270 (2018).

49. G. Auzias, O. Coulon, and A. Brovelli, "MarsAtlas: a cortical parcellation atlas for functional mapping," Hum. Brain Mapp. 37(4), 15731592 (2016).

50. MeCa, "MarsAtlas: cortical parcellation model," 2018, https://mecabrain.org/software/marsatlas/.

51. G. E. Strangman, Q. Zhang, and Z. Li, "Scalp and skull influence on near infrared photon propagation in the Colin27 brain template," Neuroimage 85(Pt 1), 136-149 (2014).

52. S. K. Sharma et al., "Dose response effects of $810 \mathrm{~nm}$ laser light on mouse primary cortical neurons," Lasers Surg. Med. 43(8), 851-859 (2011).

Paolo Cassano is assistant professor of psychiatry at Harvard Medical School and director of photobiomodulation at the Massachusetts General Hospital (MGH) Depression and Clinical Research Program and principal investigator at the MGH Center for Anxiety and Traumatic Stress Disorders. Since 2009, he has led multiple studies on transcranial photobiomodulation with nearinfrared light for anxiety and mood disorders, including translational studies aimed at discovering the mechanisms of action of photobiomodulation.

Anh Phong Tran is a doctoral candidate in chemical engineering at Northeastern University. He previously received a BS in chemical engineering from Tufts University. His research interests are in modeling and simulation in the areas of medical imaging, immunology, and control theory.

Michael R. Hamblin is an associate professor at Harvard Medical School. He works in photodynamic therapy and photobiomodulation. 
He has published over 447 peer-reviewed articles, is editor-inchief of "Photobiomodulation, Photomedicine, and Laser Surgery." With an h-factor 95, he has authored/edited 24 textbooks on PDT and photomedicine. Awards include the first Endre Mester Lifetime Achievement Award from NAALT, an Outstanding Career award from Dose Response Society, and the first Ali Javan Award in Basic Research from the World Association for Laser Therapy. He is a fellow of SPIE.

Yaoshen Yuan is a doctoral candidate at Northeastern University. He received his BE degree from Southeast University, China, in 2014 and MSE from Tufts University in 2016. His research interests include Monte Carlo simulation for photon transport, GPU algorithm enhancement and signal processing
Qianqian Fang is currently an assistant professor in the Bioengineering Department, Northeastern University, Boston, USA He received his $\mathrm{PhD}$ from Dartmouth College in 2005. He then joined Massachusetts General Hospital and became an assistant professor in 2012, before he joined Northeastern University in 2015. His research interests include translational medical imaging systems, low-cost point-of-care devices for resource-limited regions, and high performance computing tools to facilitate the development of nextgeneration imaging platforms.

Biographies of the other authors are not available. 